\title{
Riding the Web Evolution: from Egoism to Altruism
}

\author{
Marco Roccetti, Stefano Ferretti, \\ Claudio E. Palazzi, Paola Salomoni \\ Dip. di Scienze dell'Informazione, Università di Bologna \\ Mura Anteo Zamboni 7, 40127 Bologna, Italy \\ \{roccetti, sferrett, cpalazzi, salomoni\}@cs.unibo.it
}

\author{
Marco Furini \\ Dipartimento di Informatica \\ Università del Piemonte Orientale \\ Via Bellini 25/G, 15100 Alessandria, Italy \\ marco.furini@mfn.unipmn.it
}

\begin{abstract}
Internet and the latest correlated technologies, such as Web 2.0, represent inventions that have changed the world with their social contribution. Yet, digital divide and cyberbalkanization are Internet's discriminatory phenomena well known by field experts. In addition, we want now to raise the attention on the actual employment of these technologies: the vast potential within the Internet is currently dispersed in millions of applications devoted to frivolous and egocentric utilizations. Being (almost) the only use people have made of the most pervasive communication system ever made, this represents an unacceptable waste of opportunities. To revert this trend, we propose a novel utilization paradigm of Internet technologies which is aimed at liberating part of resources that are captured but not used by egocentric applications to give them back to a possible common (and altruistic) use.
\end{abstract}

\section{INTRODUCTION}

Internet represents the epochal invention that has allowed the outbreak of the speed, the amplitude, and the distance of Information access. Web 2.0, then, has made data independent from their generators and hence available to be employed for the creation of an incredible amount of new integrated services. It is hence not surprising that the stereotype of the Internet is that of an almost omnipotent instrument, able to connect in a fraction of a second millions of people and an immense amount of data; an instrument able to create new spaces of freedom giving voice to any opinion, to make us save time and money, to assist us in our investments, choices, even medical cures and interpersonal relationships.

Unfortunately, even if widely diffused, this vision of the Internet as a universally aggregating instrument is deeply distorted. Main critics moved by field experts (computer scientists, but also sociologists and philosophers) regard both the external closure toward disadvantaged social categories and the reciprocal inner closure among groups of users. In the first case we are talking about digital divide, which relates to the impossibility for people with physical, economic, cultural, or geographic limitations to have access to Internet resources. In the second case, we are referring to the cyber-balkanization phenomenon, which indicates the division of users into groups that do not interact with each other.

Beyond these critical notes, another important reason of disappointment has not yet been considered. Indeed, by analyzing the use made of Web 2.0 and, in general, of the Internet, it is easy to notice that nearly all the vast potential of this technology is dissipated in expressions of selfishness, without practical advantages for the rest of the society. Consider, for instance, most popular applications: YouTube, MySpace, and SecondLife. The rationale behind the success of all these initiatives can always be found in egocentric motivations, such as monetary gain, self-celebration, or the escape from a disappointing truth. It is hence necessary to redesign the utilization paradigm of Web 2.0 and Internet so as to give back their resources, at least partially, to a public and shared employment. Although, the personal use of Internet has not to be eliminated, the current deployment of communication resources constitutes an inexcusable waste if aimed only at supporting a frivolous and egoistic use.

Therefore, in this paper we propose an innovative utilization paradigm aimed at extending Internet's benefits to every component of our society through the re-use of (part of) computational and communication resources employed for "selfish" purposes. We also discuss on how this can be achieved via the creation of a communication platform that includes mechanisms for resource meta-tagging and quick recovering, and for efficiently sharing correlated information even in unstructured contexts (e.g., mobile ad-hoc networks).

It is time for an altruistic alternative to emerge (we could call it altruistic networking) and serve an ideal of social solidarity. If Web 2.0 was based on the saying "yield knowledge and you will acquire power," the motto at the base of our proposal goes beyond: "yield resources, you will make the good of the society, and hence your own good."

The rest of the paper is organized as follows. In Section II we provide a critic overview of current employment of the latest Internet-based technology. Section III represents the manifesto of our proposal, whereas Section IV describes characteristics that the communication platform has to possess to foster altruistic utilizations. Finally, in Section V conclusions are drawn.

\section{WWW: WORLD WIDE WASTE}

Stereotypes describe the Internet as a democratizing agent that creates new spaces of freedom and benefits for everybody. These stereotypes do not correspond to the reality: the current Information Society is characterized by an unfair access to new technologies, thus creating development inequalities [1]. Main causes of this gap have to be searched in: 
- educational marginalization, illiteracy denies the possibility to access and to generate information;

- structure marginalization, insufficient availability of resources can deny the use of a certain technology;

- technological marginalization, the use of more and more sophisticated technologies involves a continuous technological modernization that can be problematic for economic-cultural reasons;

- social marginalization, people affected by physical disabilities are excluded from the use of many technologies;

- economical marginalization, technology may be too expensive for certain people, thus excluding them from its use.

Not only, popular online applications are utilized almost exclusively for leisure or other personal motivations (e.g., work). Think, for instance, to games, chat, e-mail, and even P2P-based file sharing programs. No serious benefit for our society or for its weakest components has yet been extracted from the available powerful communication technology. Considering the immense benefits that this technology could bring to the whole society, its current employment represents an unacceptable waste: a World Wide Waste.

Indeed, even focusing on the Web, the situation does not change. The Web has been defined by its own creator as "more of a social creation than a technical one" [2] and even the Web 2.0 is definitely following this track [3]. Instead, abandoned to the market, even this technology has become a source of social/cultural gap and an emanation of a multitude of individual egos $[4,5]$.

Consider as an example, Google Maps, the service through which Google yields its maps to anybody. New applications can thus be created by third parties that can take advantage of data that otherwise would not be accessible by them. However, the real motivation at the base of Google's behavior is not generosity; rather; it is the fact that through this process Google earns visibility and sold advertisement spaces.

Another well known example is represented by Wikipedia, which defines itself as "the biggest multilingual free-content encyclopedia on the Internet." Wikipedia is written collaboratively by volunteers and its success depends mainly on its inclusiveness, intended as the number of users that is actively involved in the writing of its contents. On the other hand, in spite of its universality, Wikipedia excludes a very large number of users. In particular, Wikipedia is not a completely inclusive instrument with respect to people with disabilities as it does not follow main guidelines and norms for Web accessibility, neither from a reader's standpoint, nor from an author's one.

The last example that we cite is YouTube, "a service for online video streaming that allows anyone to visualize and share videos uploaded by members." Even the extraordinary success of this system is based on the enormous dimension of its community. However, in this case the inclusive force of YouTube resides in the popularity that the author obtains when its video enters in the top ten of most downloaded contents. In most cases, people contributes with their videos moved by a self-promotion aim. Recent news about the fact that video uploader will receive part of the advertisement revenues strengthen even more this approach. Moreover, YouTube's interface does not possess accessibility characteristics and this demonstrates that the inclusive potential of the system is not completely developed. In particular, neither the production of contents in more languages nor the management of subtitles and alternative descriptions of the video are supported.

Moreover, all the aforementioned services are utilizable only by Internet users, while there does not exist mechanisms for the distribution of such contents on other platforms, e.g., the digital television system and mobile ad-hoc networks

In summary, if on one hand these examples confirm the aim for inclusiveness and for the creation of real social networks, on the other hand we notice that a great part of the society is left out of this phenomenon. In general, communities using Web 2.0 systems are not moved by social interests, rather, they interact for personal interests in which the individual ego prevails and creates a multitude of communities sharing interests which are greatly limited in scope. The will to emerge has thus created the era of egocasting.

\section{ThE MANIFESTO}

We are aimed at creating a new interpretation of the potentialities of the Internet and of its correlated technologies (e.g., Web 2.0), to increase the social solidarity and to favor the establishment of a new, altruistic Information Society. Advantages deriving from the epidemic dissemination of information must finally become easily accessible to anyone, independently from revenue, physical abilities, and computer knowledge. Not only, starting from the diffuse availability of data and resources (intended as both hardware and software resources), new applications can and must be devised in order to provide social utility to the community.

This vision can become real by redesigning the utilization paradigm of Internet and Web 2.0. The first step in this direction is to study, plan, implement, and test a communication infrastructure able to integrate the various communication platforms: computer/Internet, mobile, TV. The complete integration of these platforms is aimed at generating a global communication scenario, able to guarantee to every member of the society the free access to information in an independent way with respect to the adopted device.

The second step is that of creating new altruistic services and applications, by which resources and information disseminated over the network can be used for social purposes thanks also to their diffusion in various contexts. The idea amounts to design a platform such that even a selfish use of the technology in order to achieve personal objectives or to have fun is rerouted by a layer devoted to the management and diffusion of collective information so as to make emerge an altruistic purpose. 
Indeed, nowadays, people tend to think that means are neutral and that it is the use made of them that makes them good or evil. Instead, we are convinced that this is an ingenuous interpretation and that through an appropriate organization and management of resources it is possible to generate an abstraction of the technological platform that is resilient to pure egoistic uses. Indeed, we should not expect that users employed devices "in the right way," rather, the system has to be able to liberate part of what is captured (and not used) by the various devices and communication technologies in order to give it back to a possible common use [6]. This novel altruistic utilization paradigm of the technology can create the conditions for which, paradoxically, city traffic could aid pedestrians, people working out could support elders in difficulty, curious citizens could supply means to help first responders, and cars could help to fight pollution in town.

As representative examples, four case studies follow, showing how a careful and integrated use of new technologies permits the development of new social and altruistic applications.

\section{Example \#1 - A sight-challenged pedestrian helped by cars.}

Think of a sight-challenged pedestrian who, via his PDA (used through assistive technologies, supplied by its device), can connect to the Internet to plan a step-by-step path toward his destination. Paradoxically, vehicles that are usually a menace could instead be of help for the disabled pedestrian to carry out his movements. In fact, the traffic information created and distributed by the vehicular network in that particular city area can be collected also by the pedestrian's device so as to be used to cross the road, avoid dangers, exploit public transportation, etc.

\section{Example \#2 - An elder in difficulty helped by young sportsmen.}

Remote monitoring of sick people is an application more and more diffuse in the western world. Think of one of these remotely monitored patients that experiences a sudden and unexpected worsening of his health conditions, while walking in a park. The arrival of doctors called by the monitoring station could be not timely enough. Instead, it would be more effective to have the various intelligent devices possessed by other people in the surroundings able to receive and, in turn, diffuse the aid-request until reaching a smart telephone of a doctor in the neighborhood, who could promptly take action while waiting for the ambulance. Therefore, paradoxically, who goes jogging for just his interest, becomes an altruistic subject by putting on hand his technological resources (e.g., an upgraded iPod with communication capabilities).

\section{Example \#3 - First responders' efficiency improved by curious people passing by the emergency area.}

Consider a crisis scene in a city context, e.g., a street accident or a terrorist attack. In this scenario, it would be useful to provide rescuers with real-time information about the state of the emergency. Thanks to new technologies, it becomes possible for first responders to obtain images or videos of the emergency while still driving toward the crisis area. Devices utilized for this purpose could be webcams, which are becoming more and more widespread in our cities [7], or any other device possessed by people present in that particular moment in the emergency area (e.g., video-cameras on vehicles, camera-endowed cell phones). Data can be sent through the vehicular network directly to the vehicle of first responders, or reach them through the Internet and the cellular network. In any case, elements that are typically considered of disturbance in emergency situations (e.g., vehicles that increase the traffic and slow down the arrival of ambulances, "curious" people who typically interfere with aid operations) turn out to be of help in preparing emergency teams to face a crisis, even before reaching the destination.

\section{Example \#4 - Town's pollution diminished thanks to cars.}

Cars cause pollution; yet, through sensors, they could become active controllers of pollution levels and distribute this information to the community. Data could be made available in the Internet, or even passed to the television system. This would allow citizens to simply receive on their TV sets information that advices against going to a certain part of the town. Moreover, mechanisms of dynamic navigation could reroute vehicles so as to diminish the number of cars transiting through sensitive areas (e.g., school areas or highly polluted neighborhoods).

\section{A New Altruistic Vision in Practice}

We propose to develop a resource (i.e., data, processor, memory, video-cameras, etc.) management system that will generate an altruistic utilization of the network. Solutions have to be individuated to restitute resources captured but unused by single users to a public and common employment.

Users will still be free to generate and use applications devoted to monetary gain or to self-promotion; however, at the same time, their technological means and information patrimonies will be also available, in an anonymous and combined way, for a use devoted to social utility. Through a shared process of collection, exchange, and spread of resources (e.g., data, devices, etc.) a novel technological platform can be implemented, which will be resilient to a pure egoistic use and naturally predisposed toward a positive employment. This new technology can create conditions to take the paradoxical examples introduced in Section III and put them into practice.

\section{A. Altruistic networking: services and characteristics}

Novel approaches have to be developed to assist the communication and management of resources disseminated over the network, in order to effectively use them for common benefit; main issues and directions of investigation are highlighted in the following.

Freedom from the technological device. The envisioned technological scenario is heterogeneous and includes several platform technologies that have to coexist in an integrated way. Users should not be forced to utilize a particular device or 
technology, rather, information have to be available through different devices/technologies and accessible also by any user. Therefore, the infrastructure has to be able to manage DVB-T, DVB-H, T-DMB, DAB, GSM/EDGE, UMTS/GPRS, WLAN, H2LAN, LAN, ADSL, Bluetooth, and HomeRF, with the possibility of being integrated with future developments, such as $4 \mathrm{G}$ or UWB. Moreover, the communication infrastructure should not consider these possibilities only during the initial offering phase, but it should do so also while actually providing the service, to dynamically determine and adopt those solutions that guarantee in every moment the best available conditions, even through seamless changes of the transmitting technology $[8,9]$.

Media integration. The infrastructure should manage and integrate various technologies in a transparent way, permitting the access, the production, and the integration of information coming from a multitude of media (e.g., computer/Internet, domestic television, mobile), and making them available in several contexts. To this aim, transcoding tools are crucial to transform contents developed for a specific media typology into a format compatible with other media (e.g., think of contents developed for high resolution PC's screens that must be visualized on lower resolution screens such as television or mobile devices) [10].

Development of social applications. Through our proposed technology, applications able to act on the morphology of the society should be easily developable. Applications such as remote education, road safety, social networks, health and public emergency, management of climatic events, information, and entertainment can be beneficial for the society. For instance, think of a scenario where a user needs to retrieve an image relative to a specific area. User's request could be forwarded to the community and some mobile/fixed device in the area could be available to acquire and transmit the image. Such kind of application could be of great social benefit, allowing, for instance, first responders to get detailed information of a critical area (see Example 3 in Section III).

Service personalization. Applications should interact with users through interfaces designed keeping into account psychology and cognitive ergonomics, so as to guarantee system's accessibility and usability; without these features the design effort would be vain. As an example, textual contents must be rendered also in a sonorous way, to respond to the needs of sight-challenged or illiterate people [11]. The necessity to adapt the information to the characteristics of the device, of the technology, and of the user, requires the investigation of solutions able to produce alternative representations of the primary contents. The management of multilingual versions (regarding both textual and audial contents), subtitles also in other languages, and the possibility to provide various interpretations of texts and contents are some possible approaches that go in this direction.

Localization systems. The localization of the user or of the device is a necessary condition for many social applications such as, for instance, safe-driving that requires to know cars' position; or climatic applications, that interconnect sensors and cell-phones endowed with localization systems to provide environmental information on a certain area. Effective solutions for localizing customers and mobile devices have hence to be proposed and investigated. The use of GPS devices represents one of the possibilities. However, this type of localization is not sufficient for various reasons: energetic limits (the high energetic consumption of these devices could limit their use in contexts of limited resources) and environmental limits (the presence of physical barriers such as tunnels compromises the effectiveness of these devices). Localization through radio waves or through passive localization means such as RFIDs can be remarkably useful for the democratizing purposes of this project.

Information categorization and research. The infrastructure has to allow users to interact with the system to retrieve information in a simple and effective way, so as to transform the information in knowledge. Content classification becomes fundamental. Given the great amount and variety of contents, the simple use of classic taxonomy and methods of formal classification do not seem to be very effective. On the contrary, the idea of having users directly classifying contents is becoming more and more popular [12]. Through the class of solutions defined as folksonomy, users have the possibility to label the information without predetermined limits; the classification depends on the characteristics of the population that creates the folksonomy. Based on this concept, the development of filters able to retrieve information in a simple and effective way is hence of fundamental importance for knowledge widespread. Indeed, as folksonomies generally applied to all sort of resources will generate a huge amount of tags, it is necessary to study filtering, clustering, and gossiping mechanisms to organize them in order to facilitate resource retrieval [13-15].

\section{B. Implementation architecture}

Focusing on the logical process, the creation and distribution of the information follow the outline depicted in the Fig. 1 (noticeably, such outline does not represent the module architecture that will derive from it). In essence, users exploit and yield their resources, which are classified and "tagged" following appropriate schemes related to folksonomies (Resources Tagging in Fig. 1). Efficient communication mechanisms will be employed to permit the distribution of such information over various network typologies (Tag and Info Distribution in Fig. 1). In order to enable a correct fruition of information and contents, opportune modalities of data management will be developed, depending on the platform and on the context. Considering the high amount of tags present in the system, mechanisms for filtering and clustering will be used to organize tags and select contents of interest (Integrated Platform for Content Presentation in Fig. 1). Such functionalities will be the base for the construction of social applications (Social Applications in Fig. 1). 


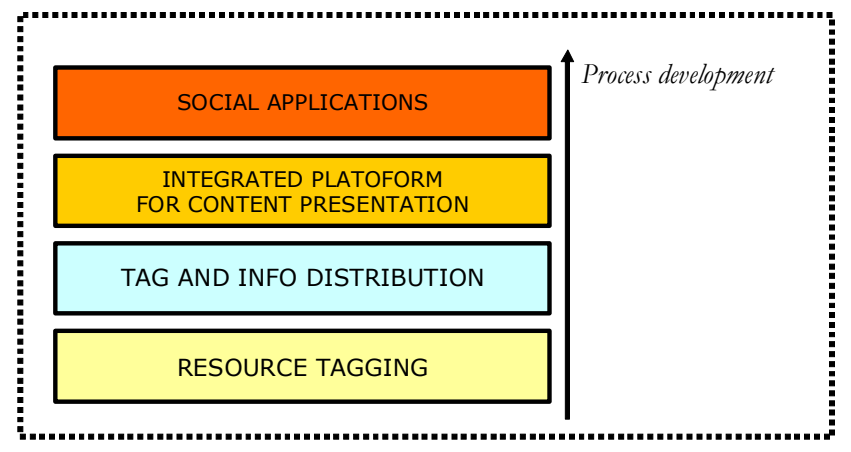

Figure 1. Information creation and distribution: logical flow.

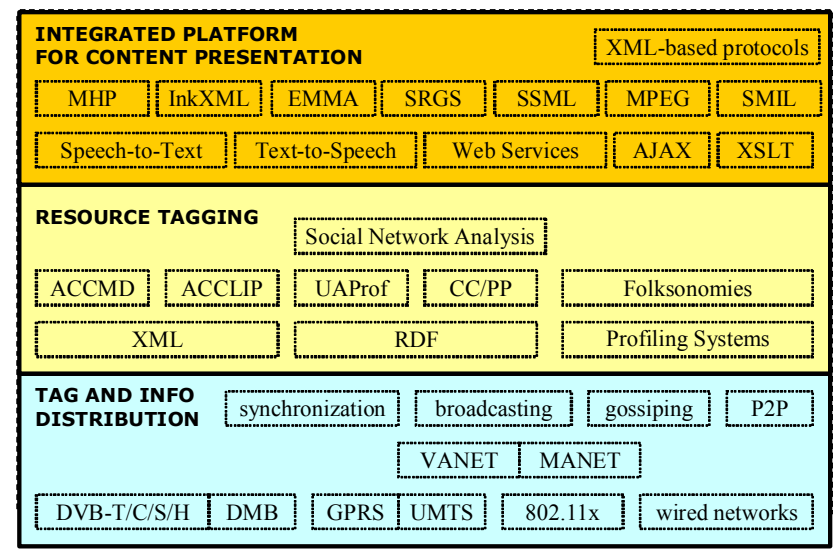

Figure 2. Existing technologies for contents management and distribution that will be integrated into the new "altruistic platform."

To put into practice the aforementioned logical functionalities, it is necessary to use, develop, modify, and integrate several existing technologies for content management and distribution (see Fig. 2). Considering a top down approach, technologies to treat and codify the information become fundamental to guarantee a social- and altruistic-devoted production, presentation, modification, and enrichment of contents (Integrated Platform for Content Presentation in Fig. 2). More in detail, it becomes important to be able to manage all meta-information characterizing contents to facilitate their recovery and management. To this aim, methodologies for assigning meta-data and profiles have to be recovered and widened (Resource Tagging in Fig. 2. Finally, the distribution of meta-data and contents have to be handled by effective communication mechanisms (Tag and Info Distribution in Fig. 2, able to diffuse such data through all network typologies, e.g., cellular networks, television networks, classic Internet networks, ad-hoc networks, mobile networks (both vehicular and pedestrian); this way, a unique and consistent social network will be created.

\section{CONCLUSION}

Internet possesses an incredible social potential as demonstrated by the recent evolution toward Web 2.0. Yet, this social potential is far from being effectively developed. Digital divide and cyber-balkanization are well known phenomena that renders these technologies discriminating agents rather than democratizing ones.

Moreover, we have here pointed out how the Internet and even Web 2.0 are mostly exploited for egocentric purposes such as monetary gain, self-promotion, personal leisure, etc. Considering the immense benefits that these instruments could bring to the whole society, their current utilization represents an unacceptable waste.

Instead, we propose a new utilization paradigm of Internetbased technologies aimed at liberating part of computational and communication resources captured, but unemployed, by "selfish" applications and re-using them for social purposes. We have described possible examples of employment of such system and outlined how resorting to (hardware and software) resource meta-tagging can be exploited to this aim.

Finally, we have indicated several investigation directions whose efficient solutions would factually contribute to the creation of the envisioned altruistic networking system, able to restitute Internet and Web technologies to an ideal of social solidarity: "yield resources, you will make the good of the society, and hence your own good."

\section{REFERENCES}

[1] K. S. Gill, Knowledge and the Post-industrial society. Information Society, K. S. Gill (ed.), Springer, New York, 1996.

[2] T. Berners-Lee, and M. Fischetti, Weaving the Web. The original design and ultimate destiny of the World Wide Web, by its inventor. Harper San Francisco, 1999.

[3] P. McFedries, "Technically speaking: social-ism," IEEE Spectrum Magazine, Vol. 44, No. 4, pp. 68-68, April 2007.

[4] M. Morcellini, Comunicazione/globalizzazione. Le architetture sociali del network space. Il Dubbio, Lithos Ed., 2002.

[5] P. J. Tichenor, G. A. Donohue, and C. N. Olien, "Mass media flow and differential growth in knowledge," Public Opinion Quarterly, No. 34, pp. $159-170,1970$.

[6] L. Gallino, Tecnologia e democrazia. Conoscenze tecniche e scientifiche come beni pubblici. Giulio Einaudi Editore, Torino, 2007.

[7] M. Roccetti, M. Gerla, C. E. Palazzi, S. Ferretti, and G. Pau, "First responders' crystal ball: how to scry the emergency from a remote vehicle," IEEE NetCri07-IPCCC07, New Orleans, LA, USA, April 2007.

[8] C. Mascolo, L. Capra, and W. Emmerich, "Middleware for mobile computing," Networking 2002 Tutorial Papers, Springer-Verlag LNCS 2497, Pisa, Italy, May 2002.

[9] G.-C. Roman, G.P. Picco, and A.L. Murphy, "Software engineering for mobility: a roadmap," Int. Conf. on Software Engineering (ICSE00), Limerick, Ireland, June 2000.

[10] S. Ferretti, M. Roccetti, C. E. Palazzi, "Web content search and adaptation for IDTV: one step forward in the mediamorphosis process toward Personal-TV," Advances in Multimedia, Hindawi Publishing Corporation, April 2007.

[11] C. Asakawa, H. Takagi, "Annotation-based transcoding for nonvisual web access," Int. ACM SIGCAPH Conf. on Assistive Technologies (ASSETS 2000), Washington, DC, USA, November 2000

[12] P. Mika, "Ontologies are us: A unified model of social networks and semantics," Web Semantics: Science, Services and Agents on the World Wide Web, Elsevier Science Publishers, Vol. 5, No. 1, pp. 5-15, 2007.

[13] J.C. Paolillo, S. Penumarthy, "The social structure of tagging Internet video on del.icio.us," 40th Annual Hawaii International Conference on System Sciences (HICSS'07), Manoa, HI, USA, 2007.

[14] T. F. Gonzalez, "Clustering to minimize the maximum intercluster distance," Theoretical Computer Science, Vol 38(2/3), pp.293-306, 1985.

[15] H. Balakrishnan, M. F. Kaashoek, D. Karger, "R. Morris, and I. Stoica, Looking up data in P2P systems," Communications of the ACM, Vol. 46, No. 2, pp. 43-48, February 2003. 